\title{
Vesicular Neurotransmitter Transporter Expression in Developing Postnatal Rodent Retina: GABA and Glycine Precede Glutamate
}

\author{
Juliette Johnson, ${ }^{1,2}$ Ning Tian, ${ }^{5}$ Matthew S. Caywood, ${ }^{2}$ Richard J. Reimer, ${ }^{4}$ Robert H. Edwards,${ }^{2,3}$ and \\ David R. Copenhagen ${ }^{1,2}$ \\ Departments of ${ }^{1}$ Ophthalmology, ${ }^{2}$ Physiology, and ${ }^{3}$ Neurology, University of California School of Medicine, San Francisco, California 94143, ${ }^{4}$ Department of \\ Neurology and Neurological Sciences, Stanford University School of Medicine, Stanford, California 94305-5489, and 5Department of Ophthalmology and \\ Visual Science, Yale University School of Medicine, New Haven, Connecticut 06520
}

Vesicular transporters regulate the amount and type of neurotransmitter sequestered into synaptic vesicles and, hence, the kind of signal transmitted to postsynaptic neurons. Glutamate is the prominent excitatory neurotransmitter in retina; GABA and glycine are the main inhibitory neurotransmitters. Little is known about the ontogeny of vesicular neurotransmission in retina. We investigated expression of glutamatergic [vesicular glutamate transporter 1 (VGLUT1)] and GABA/glycinergic [vesicular GABA/glycine transporter (VGAT)] vesicular transporters in postnatal retina. VGLUT1 labels glutamatergic synapses. VGLUT1 and synaptic vesicle 2 colocalized to photoreceptor terminals. VGLUT1 colocalized with PKC to rod bipolar terminals and to $\mathrm{ON}$ bipolar terminals in metabotropic glutamate receptor $6^{+/-}$ mice. Developmentally, VGAT expression precedes VGLUT1. In rat and mouse retina, VGAT occurred in the inner retina by postnatal day 1 (P1). In rat retina, VGLUT1 was in the outer retina by P5-P7 and the inner retina by P7. In the mouse retina, VGLUT1 expression was in the outer retina by $\mathrm{P} 3$ and the inner retina by $\mathrm{P} 5$. Both rat and mouse retina had an adult pattern of VGLUT1 expression by P14. VGLUT1 expression precedes ribbon synapses, which are first observed in the inner retina at P11 (Fisher, 1979) in mouse and P13 (Horsburgh and Sefton, 1987) in rat. The ribbon synapse marker RIBEYE was not detected in inner retina of P5 or P7 rat. Spontaneous EPSCs in mouse ganglion cells were recorded as early as P7. Together, these findings indicate that vesicular GABA and glycine transmission precedes vesicular glutamate transmission in developing rodent retina. Furthermore, vesicular glutamate transmission likely occurs before ribbon synapse formation in the inner retina.

Key words: GABA; glycine; glutamate; retina; VGLUT1; VGAT

\section{Introduction}

Synaptic transmission depends on the controlled release of synaptic vesicles containing a neurotransmitter. Vesicular transporters regulate the uptake and type of neurotransmitter sequestered into synaptic vesicles and, therefore, the amount and type of neurotransmitter released (Pothos et al., 2000). Recently, vesicular transporters for the primary excitatory neurotransmitter glutamate and the major inhibitory neurotransmitters GABA and glycine have been identified as vesicular glutamate transporter 1 (VGLUT1) and vesicular GABA/glycine transporter (VGAT), respectively (McIntire et al., 1997; Bellocchio et al., 2000; Takamori et al., 2000a).

VGLUT1 transports glutamate with the same kinetics as that reported for vesicular glutamate transport (Bellocchio et al., 2000) and is exclusively expressed in synaptic vesicles of glutamatergic terminals in the CNS (Bellocchio et al., 1998). Further-

Received Sept. 12, 2002; revised 0ct. 24, 2002; accepted 0ct. 25, 2002.

This work was supported primarily by National Institutes of Health grants (D.R.C. and R.H.E). Additional support was provided by a National Eye Institute core grant, That Man May See (D.R.C), and Research to Prevent Blindness (D.R.C). We thank Dr. René Renteria and Dr. David Krizaj for valuable discussions during the course of this study, Dr. Thomas C. Südhof for the RIBEYE antibody, and Edwin Dumlao for technical assistance.

Correspondence should be addressed to Dr. Juliette Johnson, Department of Ophthalmology, University of California, San Francisco, School of Medicine, 10 Kirkham Street, Room K-140, San Francisco, CA 94143-0730. E-mail: cjj@itsa.ucsf.edu.

Copyright $\odot 2003$ Society for Neuroscience $\quad 0270-6474 / 03 / 230518-12 \$ 15.00 / 0$ more, VGLUT1 shares sequence similarity with a Caenorhabditis elegans mutation, EAT-4, which causes disrupted presynaptic glutamatergic transmission (Lee et al., 1999). Taken together, these studies provide strong evidence that VGLUT1 acts as a vesicular glutamate transporter.

VGAT transports GABA with characteristics similar to those reported for vesicular GABAergic transport (McIntire et al., 1997). VGAT antibody immunolabels GABAergic synaptic vesicles in inhibitory terminals, whereas glutamatergic nerve terminals are not immunoreactive (Chaudhry et al., 1998). In addition, VGAT immunolabels glycinergic nerve terminals, suggesting that VGAT transports glycine in addition to GABA (Chaudhry et al., 1998). Transport studies confirm that VGAT transports both inhibitory neurotransmitters glycine and GABA (McIntire et al., 1997).

The expression of specific vesicular transporters directly determines phenotype. GABAergic neurons transfected with VGLUT1 are transformed into glutamatergic neurons (Takamori et al., 2000a), and PC12 cells transfected with VGAT display vesicular transport of GABA (McIntire et al., 1997). A subpopulation of synaptic vesicles immunoisolated from rat brain using VGAT antibody exclusively transport GABA, indicating that VGAT expression confers a GABAergic phenotype (Takamori et al., 2000b).

VGAT expression has been investigated in several mammalian 

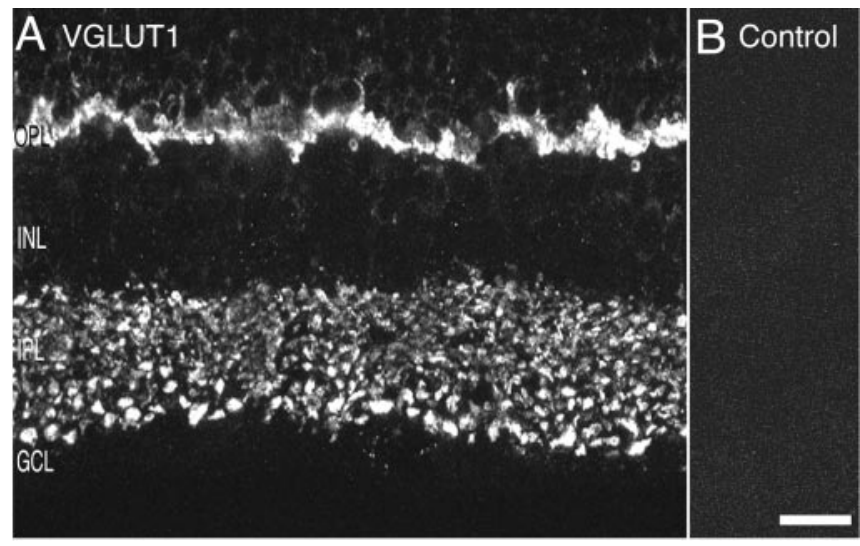

Figure 1. VGLUT1 immunoreactivity localizes to the synaptic layers of retina. Photomicrographs of vertical sections through rat retina are shown. $A$, Confocal fluorescence micrograph of VGLUT1 immunolabeling. VGLUT1 expression is confined to the outer and inner plexiform layers where vesicular glutamate transmission occurs. In the OPL, there is intense VGLUT1 immunolabeling. Labeling is absent from the ONL. Discrete and punctate immunostaining was observed across the full extent of the IPL, with larger puncta observed in the inner portion of the IPL near the $\mathrm{GCL}$. $B$, There was no immunostaining observed when primary antibody was preabsorbed with VGLUT1 fusion protein. Scale bar, $15 \mu \mathrm{m}$.

retinas, including rat, mouse, rabbit, and monkey. VGAT localized to the outer plexiform layer (OPL) and inner plexiform layer (IPL), with weak immunostaining of cell bodies in the inner nuclear layer (INL) and ganglion cell layer (GCL; Haverkamp et al., 2000; Cueva et al., 2002). Staining in the OPL colocalized with horizontal cell markers, and VGAT immunostained horizontal dendritic processes in monkey retina (Haverkamp et al., 2000) and isolated rabbit type A and B horizontal cells (Cueva et al., 2002).

Despite detailed morphological studies of retinal synaptic architecture in developing retina, little is known about the ontogeny of vesicular glutamatergic and GABA/glycinergic synaptic neurotransmission. Recent identification of the vesicular transporters for glutamate and GABA and glycine confers a novel way to determine the onset of vesicular transmission in postnatal retina. We investigated expression of vesicular transporters for glutamate and GABA and glycine in mouse and rat retina. We also studied spontaneous excitatory and inhibitory synaptic inputs to retinal ganglion cells in young mouse retina. Spontaneous EPSCs (sEPSCs) in ganglion cells are present as early as postnatal day 7 (P7). VGLUT1 expression is present before observation of ribbons in the inner retina of mouse (Fisher, 1979; Bachman and Balkema, 1993) and rat (Horsburgh and Sefton, 1987). Our findings indicate that VGAT expression precedes VGLUT1, and that vesicular glutamate transmission occurs before the establishment of ribbon synapses.

\section{Materials and Methods}

Animals and tissue preparation for immunohistochemical experiments. Long-Evans rats (Simonsen, Gilroy, CA) at P1-P40 and adult were used. C57BL/6 mice at P1-P20 and adult (B and K Universal, Fremont, CA) were used. In addition, metabotropic glutamate receptor 6 (mGluR6) $^{+/-}$mice that express lacZ in ON bipolar cells were used (Tagawa et al., 1999). Animals were fed and housed under a $12 \mathrm{hr}$ light/dark schedule. All procedures were approved by the Committee on Animal Research, University of California, San Francisco, and were in accordance with National Institutes of Health guidelines. For the developmental studies, littermates and identical fixation protocols with parallel immunostaining were used. Rats were killed by $\mathrm{CO}_{2}$ asphyxiation followed by cervical dislocation. Young rats and mice (P1-P5) were killed by
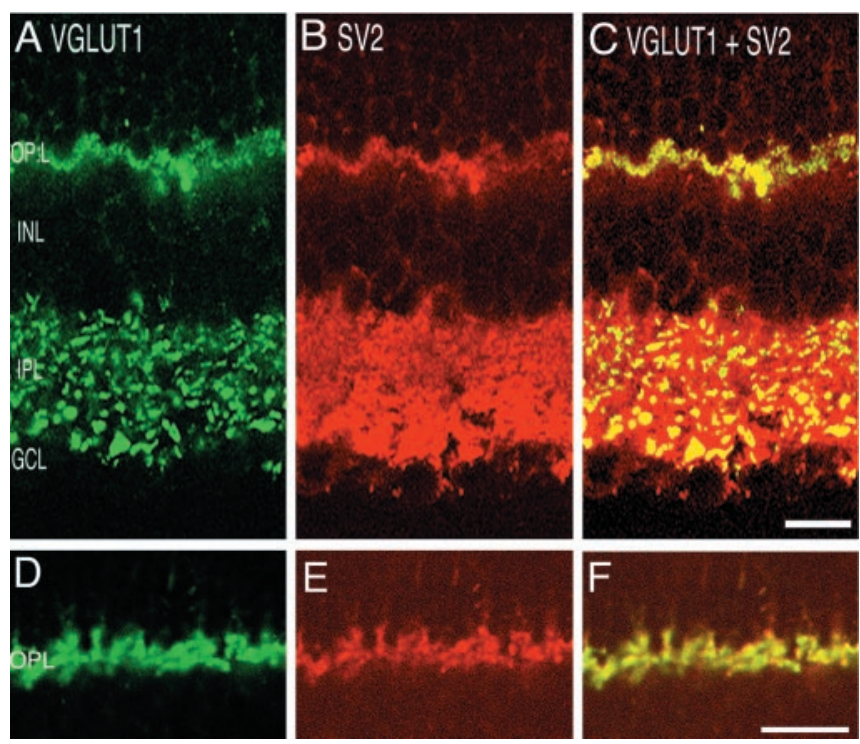

Figure 2. VGLUT1 colocalizes with the presynaptic protein SV2. Confocal fluorescence micrographs of a vertical section of rat retina are shown. $A, D$, VGLUT1; $B, E, S V 2 ; C, F$, overlay of VGLUT1 and SV2. Yellow indicates colocalization. Nearly complete colocalization occurs in the OPL, indicating expression of VGLUT1 in photoreceptor terminals. In the IPL, SV2 immunoreactivity is more widespread than VGLUT1, suggesting that VGLUT1 is expressed in a subset of synapses in the IPL. Scale bars: $A-C, 15 \mu \mathrm{m} ; D-F, 20 \mu \mathrm{m}$.

decapitation. Mice were killed by cervical dislocation. The eye was removed; the cornea was perforated with a razor blade; and the entire eye was immediately immersed in $4 \%$ (w/v) paraformaldehyde (PFA) in 0.1 $\mathrm{M}$ phosphate buffer ( $\mathrm{PB})$. In some experiments, the anterior part of the eye including the cornea and lens was first carefully removed before
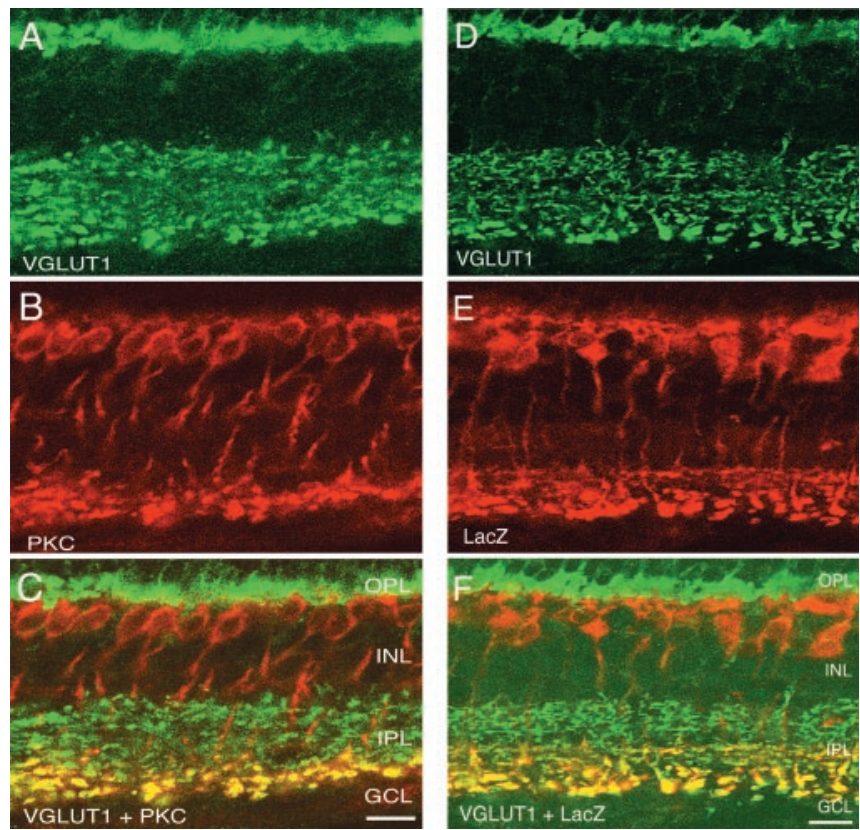

Figure 3. VGLUT1 is expressed in both rod and cone ON bipolars. Confocal fluorescent micrographs of a vertical section of rat retina are shown. $A$, VGLUT1; $B$, PKC; $C$, overlay of VGLUT1 and PKC. Yellow indicates colocalization. VGLUT1 and PKC immunoreactivities overlap in punctate structures located at the inner region of the IPL, consistent with expression of VGLUT1 in rod bipolar cell terminals. Staining was performed in retina from $\mathrm{mGluR6}^{+/-}$transgenic mouse in which LacZ is generated in the $0 \mathrm{~N}$ bipolar cells. D, VGLUT1 staining; $E$, LacZ antibody staining; $F$, overlay revealing extensive overlap in the inner half of the IPL, indicating that VGLUT1 is expressed in both rod and cone $\mathrm{ON}$ bipolars. Scale bar, $15 \mu \mathrm{m}$. 

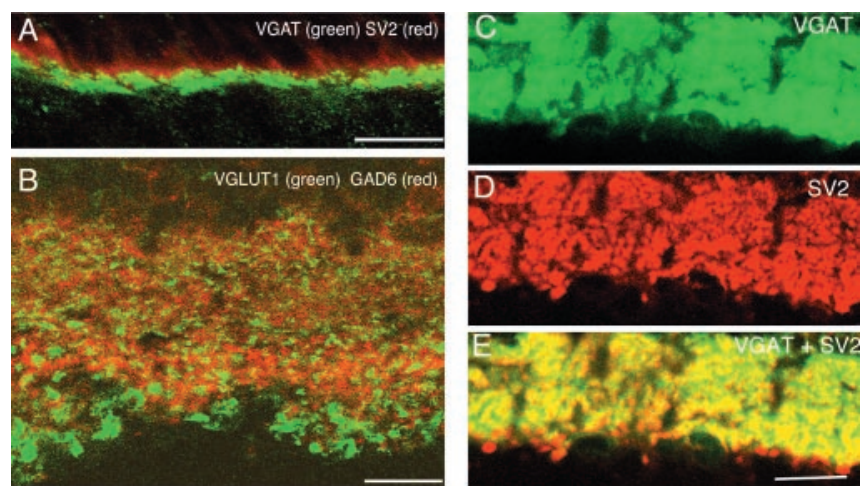

Figure 4. VGLUT1 is not expressed in GABAergic neurons. Confocal fluorescence micrographs of vertical sections of rat retina are shown. $A$, In the OPL, VGAT ( green) does not colocalize with SV2 (red), a maker of photoreceptor terminals, which express VGLUT1. B, In the inner retina, VGLUT1 ( green) and GAD6 (red), a marker of GABAergic synapses, do not colocalize. VGAT and SV2 colocalize to a subset of synapses in the IPL. Vertical sections of rat retina are shown. C, VGAT immunolabeling in the IPL; D, SV2 colocalizing $(E)$ to a subset of GABAergic synapses in the IPL. Yellow indicates colocalization. Scale bars: $A, B, 15 \mu \mathrm{m} ; C-E, 20 \mu \mathrm{m}$.

immersing the entire posterior eyecup in fixative. The eye was fixed in PFA in $0.1 \mathrm{M} \mathrm{PB}$ for $30-90 \mathrm{~min}$ at $4^{\circ} \mathrm{C}$. The eye was then stored overnight in $25 \%$ sucrose plus $0.01 \%$ sodium azide in $0.1 \mathrm{M} \mathrm{PB}$ at $4^{\circ} \mathrm{C}$. The following day, the eyes were embedded in ornithine carbamyl transferase and frozen on dry ice. Sections of the retina were cut perpendicular to the vitreal surface with a cryostat at $14-16 \mu \mathrm{m}$, mounted onto Super-Frost Plus slides (Fisher Scientific, Pittsburgh, PA), and stored frozen at $-20^{\circ} \mathrm{C}$ until immunostaining.

Antibodies. A rabbit polyclonal antibody for VGLUT1 (also known as brain-specific $\mathrm{Na}^{+}$-dependent inorganic phosphate transporter) was used. This antibody was directed against the last 68 amino acids of a rat VGLUT1 fusion protein (Ni et al., 1994; Bellocchio et al., 1998). The specificity of immunostaining was evaluated by preadsorbing the antibody with $0.2 \mathrm{mg} / \mathrm{ml}$ glutathione $S$-transferase (GST)-VGLUT1 fusion protein. Immunostaining was not apparent in sections preabsorbed with GST-VGLUT1 fusion protein. As an additional control, sections were preincubated with GST or GST-differentiation-associated $\mathrm{Na}^{+}$dependent inorganic phosphate cotransporter (also known as VGLUT2; Fremeau et al., 2001), which did not alter the staining pattern. The specificity of the VGLUT1 antibody was evaluated in a previous study using Western blotting and transfected cells (Bellocchio et al., 1998). A rabbit polyclonal antibody directed against the $\mathrm{N}$-terminal region of VGAT was used, and the specificity of this antibody was determined in previous studies (Chaudhry et al., 1998). A rabbit polyclonal antibody for VGLUT2 was also used. This antibody was directed against 64 amino acids of the C terminus of VGLUT2 (Fremeau et al., 2001).

Monoclonal antibodies against the following proteins were used: $\beta$-galactosidase (Chemicon International, Temecula, CA), glutamic acid decarboxylase 6 (GAD6) (obtained from the Developmental Studies Hybridoma Bank, Department of Biological Sciences, University of Iowa, Iowa City, IA), protein kinase $\mathrm{C}(\alpha, \beta$, and $\gamma$ isoforms, clone MC5; Santa Cruz Biotechnology, Santa Cruz, CA); RIBEYE (a kind gift from Dr. Thomas C. Südhof, University of Texas Southwestern Medical Center, Dallas, TX), and synaptic vesicle 2 (SV2; developed by Kathleen Buckley and obtained from the Developmental Studies Hybridoma Bank). Primary antibodies were detected using affinity-purified goat anti-rabbit Alexa 488, goat anti-rabbit Alexa 594, goat anti-mouse Alexa 488, goat anti-mouse Alexa 594, or goat anti-mouse Alexa 633 (Molecular Probes, Eugene, OR).

Immunohistochemical procedures. Cryostat sections were washed in 0.1 M PB, incubated $12-36 \mathrm{hr}$ in primary antibody with 0.3 or $0.5 \%$ Triton $\mathrm{X}-100$ and $10 \%$ normal goat serum at $4^{\circ} \mathrm{C}$, and then washed in $0.1 \mathrm{M} \mathrm{PB}$. Sections were incubated at a 1:1000 dilution in secondary antibody with 0.3 or $0.5 \%$ Triton X-100 and $10 \%$ normal goat serum for $1.5-2 \mathrm{hr}$ at room temperature. Sections were coverslipped with Vectashield (Vector Laboratories, Burlingame, CA) to retard fading.
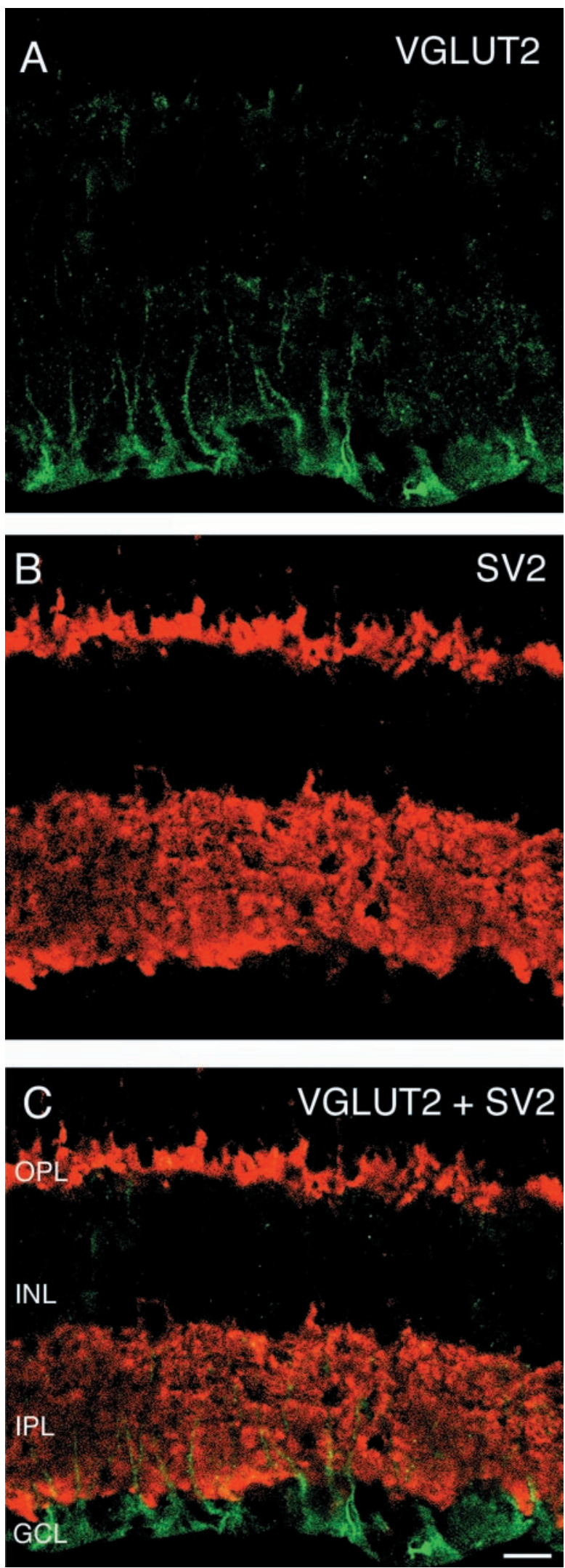

Figure 5. VGLUT2 does not colocalize with the synaptic marker SV2. Vertical sections of adult rat retina are shown. $A$, VGLUT2 immunostaining was observed in putative Müller cell processes in the inner portion of the retina, and there was also sometimes faint and diffuse immunostaining of cells bodies in the ganglion cell layer. B, SV 2 immunostaining was observed exclusively in the outer and inner plexiform layers. C, Double labeling for VGLUT2 and SV2 shows that these markers do not colocalize. Scale bar, $20 \mu \mathrm{m}$. 


\section{VGAT Developing Rat Retina}
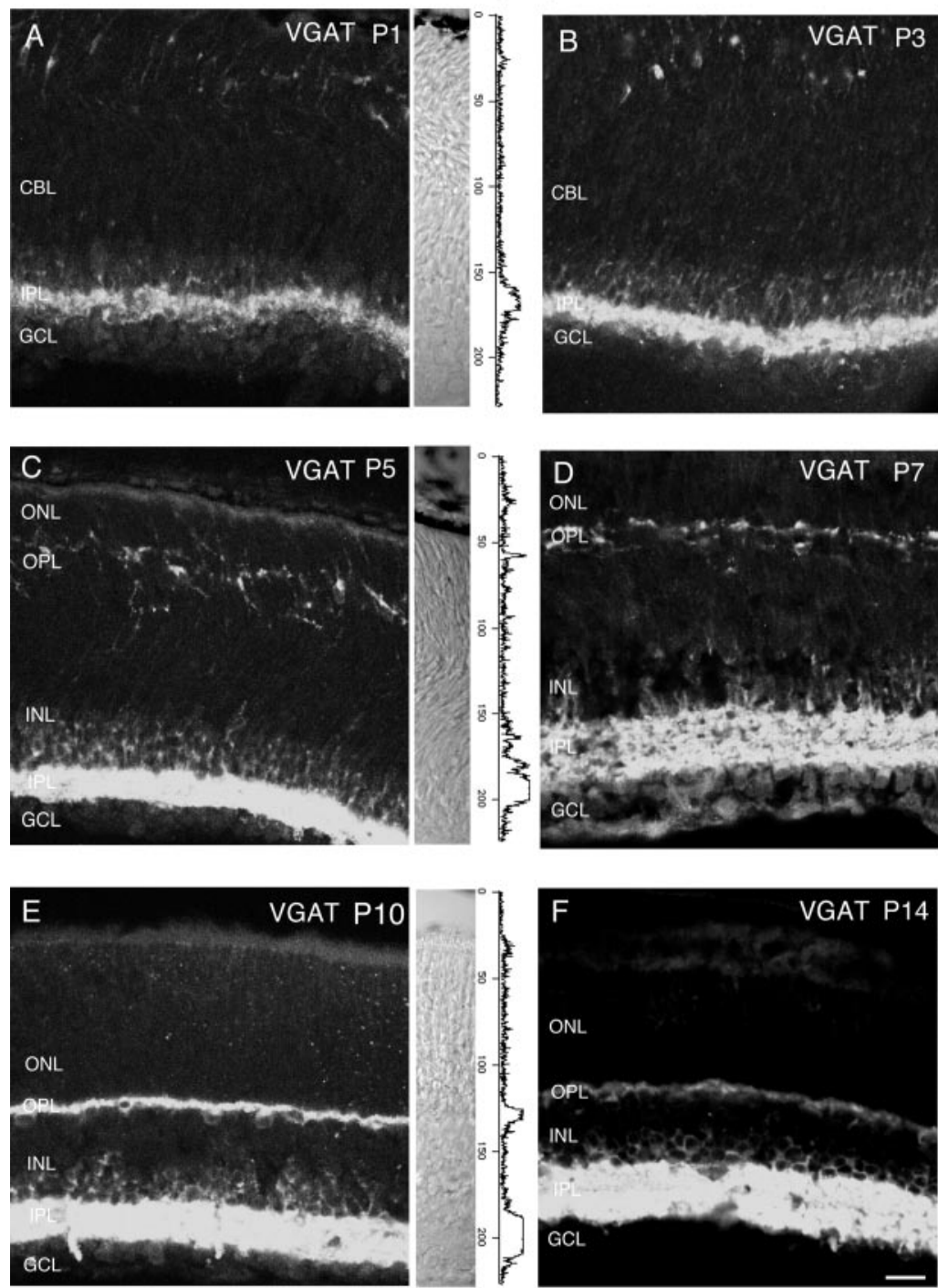

Figure 6. Vertical sections showing the postnatal development of VGAT in the rat retina. VGAT was present in the inner retina by P1. Retinal layers are shown with Nomarski optics adjacent to the micrographs. Intensity profiles are also included for each fluorescent image. VGAT immunolabeling was examined at the following ages: $A, P 1 ; B, P 3 ; C, P 5 ; D, P 7 ; E, P 10 ; F, P 14$. Scale bar, $20 \mu \mathrm{m}$.

Detection and image processing. Confocal laser-scanning immunofluorescent images were collected using $40 \times$ air and $63 \times$ oil objectives on a Bio-Rad (Hercules, CA) MRC 1034 confocal microscope. Confocal laserscanning Nomarski and immunofluorescent images were also obtained using $40 \times$ oil, $40 \times$ water, $63 \times$ water, and $63 \times$ oil objectives on a Zeiss (Thornwood, NY) Pascal confocal microscope. For double-labeling studies, sequential confocal scans were collected to prevent bleedthrough (Vu et al., 2000). To ensure that illumination at $488 \mathrm{~nm}$ did not excite Alexa 594- or Alexa 633-labeled sections (and vice versa), the fluorescence of Alexa 594- or Alexa 633-labeled sections was examined while illuminating at $488 \mathrm{~nm}$. Under these conditions, fluorescence was dark or diminutive.

Adobe Photoshop 5.5 (Adobe Systems, Inc., Mountain View, CA) was used to adjust the levels and curves of the confocal images and to make pseudocolor-overlay images. Zeiss confocal Pascal software was used to perform simple intensity profiles of the immunostaining in retinal sections.

Retinal slice preparation. Spontaneous excitatory and inhibitory synaptic inputs to mouse retinal ganglion cells were studied as reported previously (Tian et al., 1998; Tian and Copenhagen, 2001). C57BL/6 mice aged P7-P15 were used. Briefly, the eyes were enucleated and he-
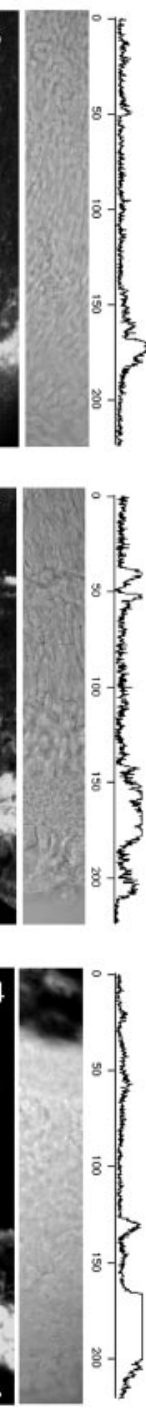

misected at the ora serata immediately after cervical dislocation. The retina was detached from the eyecup mechanically and put into oxygenated saline on a piece of Millipore (Bedford, MA) filter paper with photoreceptors facing the filter paper. The retina was cut into 250- $\mu \mathrm{m}$-thick slices. All slices were incubated continuously in oxygenated extracellular solution. Single slices were continuously perfused in the recording chamber. Tissue preparation and recordings were performed at room temperature $\left(23^{\circ} \mathrm{C}\right)$ and in normal room light. The recording chamber was mounted on the stage of an upright microscope (Axioskop; Zeiss, Oberkochen, Germany). A $40 \times$ water immersion objective lens was used for visualizing the retina and the recording pipettes. The extracellular recording solution contained (in $\mathrm{mm}$ ): $124 \mathrm{NaCl}, 2.5 \mathrm{KCl}, 2 \mathrm{CaCl}_{2}, 2 \mathrm{MgCl}_{2}, 1.25$ $\mathrm{NaH}_{2} \mathrm{PO}_{4}, 26 \mathrm{NaHCO}_{3}$, and 22 glucose, $\mathrm{pH}$ 7.35 with $95 \% \mathrm{O}_{2}$ and $5 \% \mathrm{CO}_{2}$. The pipette solution for perforated patch-clamp experiments contained (in $\mathrm{mm}$ ) $120 \mathrm{CsOH}, 110$ D-gluconic acid, $10 \mathrm{CsCl}, 5 \mathrm{CaCl}_{2}, 3 \mathrm{MgCl}_{2}, 20$ HEPES, and 30 glucose, $\mathrm{pH}$ 7.2. Perforated patch recordings were made by adding gramicidin to the pipette solution to give a final concentration of $100 \mathrm{mg} / \mathrm{ml}$ (0.2\% DMSO). All chemicals were obtained from Sigma (St. Louis, MO). The electrodes were tip-filled with a small volume (300-500 $\mu \mathrm{m}$ in length) of gramicidin-free pipette solution and then back-filled with pipette solution containing gramicidin to avoid interference of gramicidin with seal formation. Access resistance and cell conductance were monitored every 5-10 min during the entire course of each experiment. Recordings began after the access resistance reached a stable plateau $(<100 \mathrm{M} \Omega)$, which generally took 20-30 min. Membrane currents were recorded with an Axopatch 1D amplifier (Axon Instruments, Foster City, CA), and data were collected using either a Macintosh- or personal computer-based interface (ITC-16 Mac computer interface; Instrutech Corp., Great Neck, NY) run by HEKA software (Pulse + PulseFit; HEKA Elektronik). The signals were filtered at $1 \mathrm{kHz}$.

sEPSCs were separated from spontaneous IPSCs (sIPSCs) by quantification of rise time, peak amplitude, and monoexponential decay times of spontaneous events. Our previous studies have found that histograms of decay times are separated into a group of faster-decaying events and a group of slower-decaying events. The faster events are glutamatergic AMPA-mediated sEPSCs, whereas the slower events are GABA- and glycine-mediated sIPSCs (Tian et al., 1998; Tian and Copenhagen, 2001). ANOVA tests were performed to determine statistically significant events.

\section{Results}

One principal goal of this study was to test whether VGLUT1 is localized to glutamatergic neurons in the retina. Having established this expression pattern, subsequent goals addressed the developmental expression of VGLUT1 in postnatal retina. We were particularly interested in the relative time of expression of VGLUT1 compared with VGAT and whether vesicular excitatory neurotransmission depended on the establishment of ribbons in bipolar synapses. 
VGLUT1 is expressed exclusively in the inner and outer plexiform layers of retina

VGLUT1, a vesicular glutamate transporter, is expressed in adult rat and mouse retina. VGLUT1 expression is confined to theouter and inner plexiform layers where vesicular glutamate transmission occurs (Copenhagen and Jahr, 1989; Tachibana and Okada, 1991). In the OPL, between the outer nuclear layer (ONL) and INL, there was intense VGLUT1 immunolabeling in the rat (Figs. $1 A, 2 A, D)$ and mouse (Fig. 3D) retina. VGLUT1 immunolabeling was absent from the ONL, indicating lack of expression in photoreceptor cell bodies. Discrete and punctate immunostaining was observed across the full extent of the IPL with larger puncta observed in the inner portion of the IPL near the GCL (Figs. $1 A, 2 A, 3 A, D$ ). An absence of immunostaining in the INL indicates that VGLUT1 is not expressed in the cell bodies of amacrine, bipolar, horizontal, or Müller cells. Immunostaining was not observed in retinal sections that were preadsorbed with VGLUT1 GST peptide (Fig. $1 B$ ).

\section{VGLUT1 is expressed in photoreceptor} synaptic terminals

Double-labeling experiments confirmed the exclusive presynaptic localization of VGLUT1 to photoreceptor terminals in the OPL. Figure 2 shows a vertical section of rat retina that was double-labeled with antibodies against VGLUT1 (Fig. 2A,D) and SV2 (Fig. 2B,E). SV2 is a specific marker of synaptic vesicle membrane proteins (Buckley and Kelly, 1985) and is localized to photoreceptor terminals in the OPL (Rich et al., 1997; Vu et al., 2000).

There is complete overlap of VGLUT1 and SV2 labeling in the OPL (Fig. 2C,F), indicating expression of VGLUT1 in photoreceptor terminals.

VGLUT1 is expressed selectively in bipolar cell terminals in the inner plexiform layer

Punctate immunostaining for VGLUT1 was observed throughout the entire extent of the IPL (Figs. $1 A, 2 A, 3 A, D$ ). Small punctate profiles were observed in both the $\mathrm{ON}$ and OFF sublamina of the IPL (Wässle et al., 1991; Euler and Wässle, 1995), whereas larger punctate profiles were found in the inner part of the IPL adjacent to the GCL. The size and location of these punctate profiles suggest that they correspond to the synaptic terminals of $\mathrm{ON}$ and OFF cone bipolar terminals and ON rod bipolar cells, respectively.

Colocalization of SV2 and VGLUT1 expression in the IPL supports the hypothesis that VGLUT is confined to synaptic terminals (Fig. 2). In the IPL, SV2 labels both conventional and ribbon synapses belonging to amacrine and bipolar cells, respectively (Von Kriegstein et al., 1999). SV2 staining is found
VGAT Developing Mouse Retina
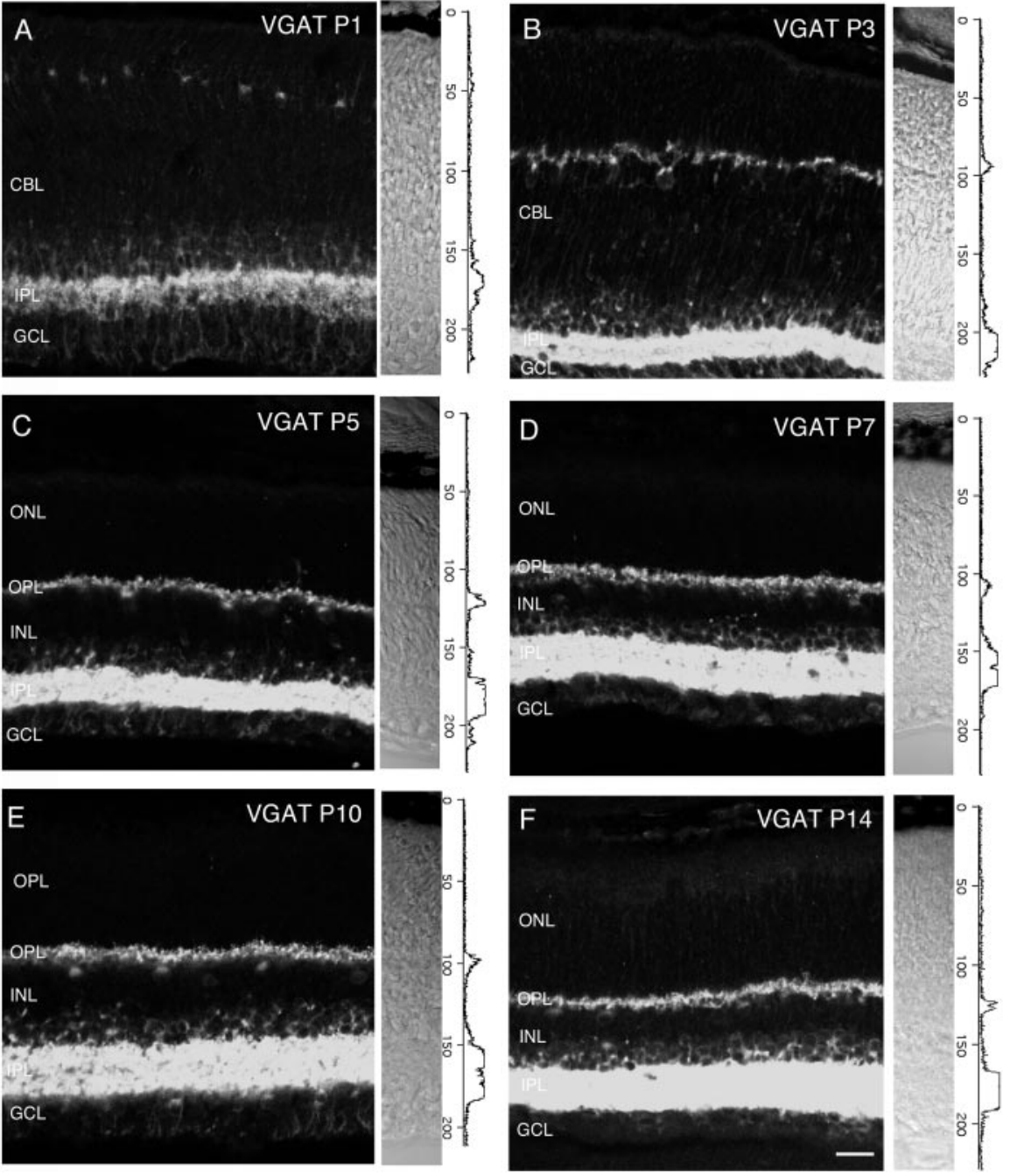

Figure 7. Vertical sections showing the postnatal development of VGAT in the mouse retina. VGAT immunostaining was observed in the inner retina by P1. Retinal layers are shown with Nomarski optics adjacent to the micrographs. Intensity profiles are also included for each fluorescent image. VGAT immunolabeling was examined at the following ages: $A, P 1 ; B, P 3 ; C, P 5 ; D, P 7 ; E$, P10; F, P14. Scale bar, $20 \mu \mathrm{m}$.

throughout the IPL (Fig. $2 B$ ). VGLUT1 is localized to discrete punctate immunostaining in the IPL (Fig. $2 A$ ). Figure $2 C$ shows that VGLUT1 immunostaining colocalizes with SV2. However, not all SV2 immunolabeling in the IPL colocalizes with VGLUT1, indicating that VGLUT1 is expressed in only a subset of the synapses in the IPL. The size, discrete appearance, and distribution of the VGLUT1 immunostained profiles observed in the IPL suggest that these correspond to bipolar cell terminals. Double labeling with GAD6, a GABAergic marker, revealed no colocalization with VGLUT1. Figure $4 B$ shows the IPL of adult rat retina immunolabeled with VGAT (green) and GAD6 (red), which do not colocalize. These results suggest that VGLUT1 is not expressed in GABAergic amacrine cell synapses.

Confirmation of VGLUT1 expression in rod bipolar cell terminals was determined by double-labeling experiments with the selective rod bipolar cell marker PKC (Greferath et al., 1990). In vertical sections of rat retina, VGLUT1 (Fig. 3A) and PKC (Fig. 3B) colocalize (Fig. 3C) to rod bipolar terminalslocated at the inner part of the IPL. These results indicate 


\section{VGLUT1 Developing Rat Retina}
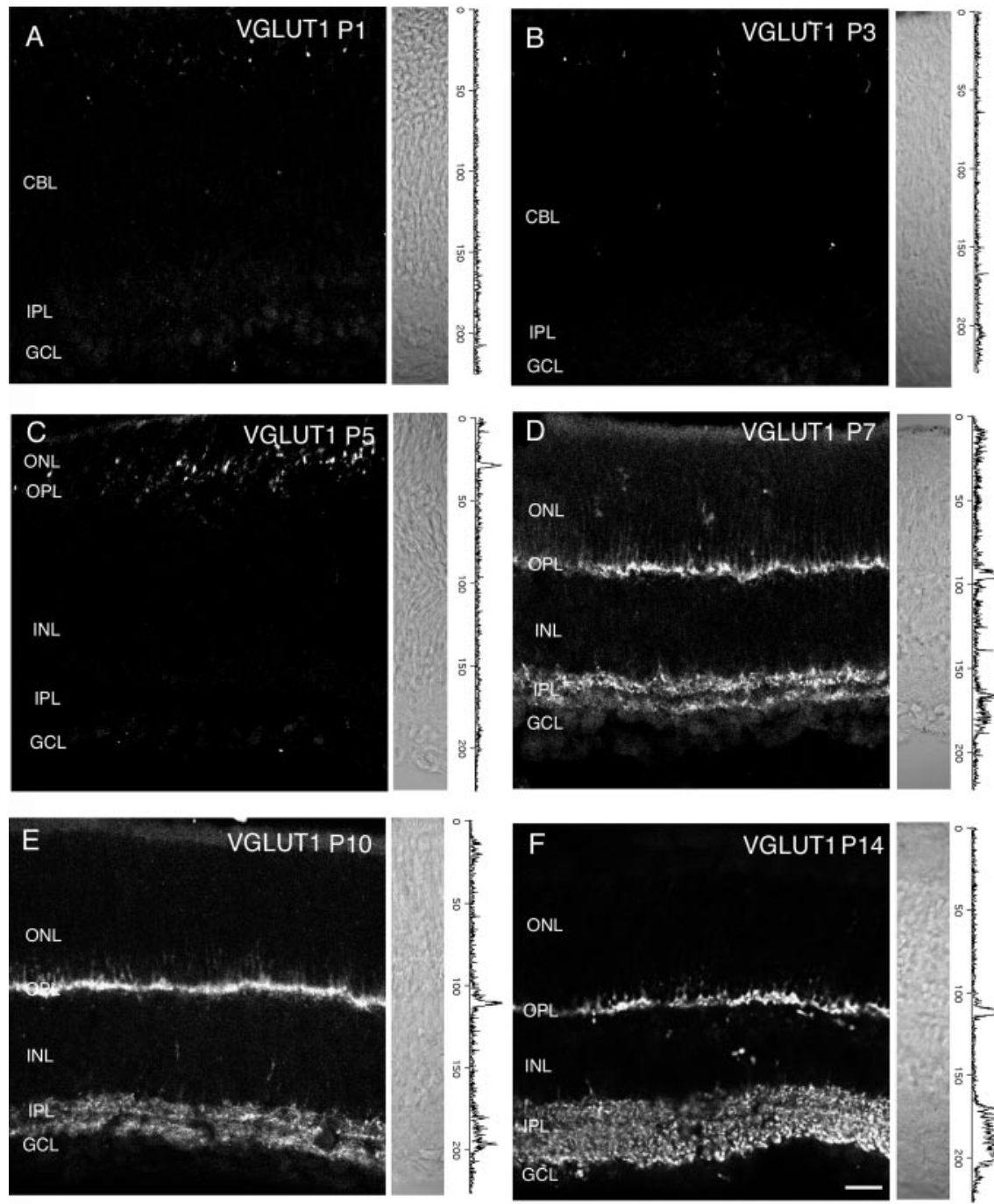

Figure 8. Vertical sections of postnatal developing rat retina immunostained for VGLUT1. VGLUT1 had delayed expression compared with VGAT. VGLUT1 was observed in the outer retina by P5-P7 and in the inner retina by P7. Retinal layers are shown with Nomarski optics adjacent to the micrographs. Intensity profiles are also included for each fluorescent image. VGLUT1 immunolabeling was examined at the following ages: $A, P 1 ; B, P 3 ; C, P 5 ; D, P 7 ; E, P 10 ; F, P 14$. Scale bar, $20 \mu \mathrm{m}$.
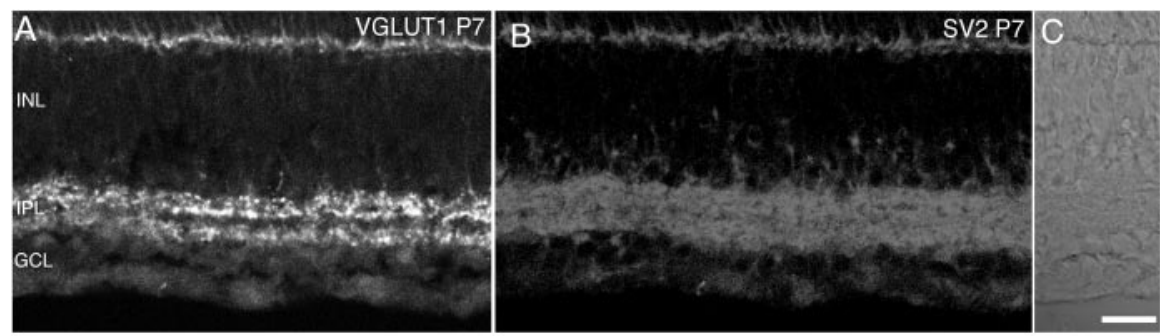

Figure 9. VGLUT1 has a selective distribution to presumptive ON and OFF strata of bipolar terminals in the IPL of P7 rat retina. Vertical sections of P7 rat retina are shown. Nomarski optics are shown adjacent to the immunofluorescence micrographs. $A$, VGLUT1 immunostaining is present in two strata in the IPL. $B$, This distinct stratification pattern is not present in SV2 immunolabeling. Scale bar, $20 \mu \mathrm{m}$.

thatVGLUT1 is expressed by ON rod bipolar terminals. We used mGluR6 ${ }^{+/-}$mice, which express LacZ in both rod and cone ON bipolar cells (Tagawa et al., 1999), to investigate expression of VGLUT1 in ON bipolar terminals. Figure 3D shows immunostaining for VGLUT1 in an mGluR6 ${ }^{+/-}$mouse,
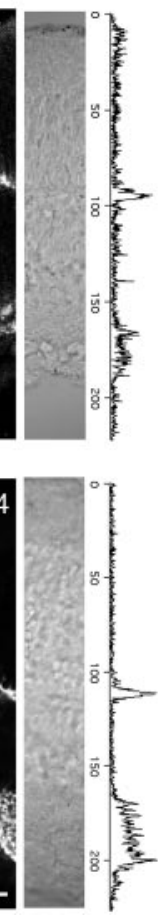

which was double-labeled with an antibody to LacZ (Fig. 3E). Figure $3 F$ shows colocalization of VGLUT1 and LacZ to both rod and cone ON bipolar terminals. Furthermore, we interpret the VGLUT1 staining pattern in the distal part of the IPL as evidence for its expression in OFF bipolar cells.

VGLUT1 is the principal vesicular glutamate transporter used by photoreceptors and bipolar cells

VGLUT2, a vesicular glutamate transporter that is closely related to VGLUT1, has recently been localized to glutamatergic synapses (Fremeau et al., 2001). In the brain, VGLUT1 and VGLUT2 show complimentary expression patterns, so that excitatory neurons that lack VGLUT1 express VGLUT2 (Fremeau et al., 2001). We investigated VGLUT2 immunostaining in the retina to establish whether photoreceptors and bipolar cells, the major glutamatergic neurons in the retina, express VGLUT2. Figure $5 A$ shows immunostaining for VGLUT2 in a vertical section of adult rat retina. VGLUT2 immunostaining was observed in putative Müller cell processes in the inner part of the retina. There was also sometimes faint and diffuse labeling of cell bodies in the GCL. Figure $5 B$ shows immunostaining for SV2, which labels synapses in the OPL and INL. Figure $5 C$ shows lack of colocalization of these two markers. These results suggest that VGLUT2 is not expressed in the glutamatergic synapses of photoreceptors or bipolar cells.

In summary, our findings show that intense VGLUT1 expression is found in the OPL and is colocalized with SV2 to photoreceptor terminals. VGLUT1 immunolabels punctate profiles throughout the distal and proximal IPL. Colocalization of VGLUT1 with PKC and with LacZ in mGluR6 ${ }^{+/-}$mice provides strong evidence that VGLUT1 is expressed in ON bipolar terminals. We also found that VGLUT1 does not colocalize with the GABAergic cell marker GAD6 in the IPL.

VGAT expression precedes VGLUT1 in developing postnatal rat and mouse retina

We determined the ontogeny of vesicular GABA and glycine and vesicular glutamate transporters by examining the time course of expression of VGAT and VGLUT1. We used SV2 expression as an internal reference, because previous experiments have extensively investigated SV2 expression in the developing rodent retina (Rich et al., 1997). VGAT and SV2 expression were present in young postnatal retina shortly after birth, whereas VGLUT1 had a delayed onset of expression. 
VGAT

As reported previously, prominent VGAT immunolabeling in adult retina was observed in the OPL and in the IPL, whereas weak immunostaining was observed in cell bodies in the INL and GCL (Figs. 4A,C-E, 6, 7) (Haverkamp et al., 2000;

Cueva et al., 2002). The pattern of VGAT immunostaining was identical in mouse and rat retina, confirming a previous report (Cueva et al., 2002). We observed weak cell body staining mainly in the INL (Figs. 6, 7), which are likely amacrine cell bodies. VGAT staining in the OPL has been reported to colocalize with horizontal cell markers (Cueva et al., 2002). In agreement with these reports, we found that the VGAT staining in the OPL did not colocalize with the photoreceptor marker SV2 (Fig. 4A). Furthermore, in doublelabeling experiments in the IPL, VGAT colocalized with a subset of SV2-labeled synapses. Figure $4 C$ shows immunolabeling for VGAT in the IPL of rat retina that was double-labeled for SV2 (Fig. 4D) and colocalization of these markers to a subset of synapses in the IPL (Fig. $4 E$ ).

Figure 6 shows VGAT immunostaining in developing postnatal rat retina. Vertical retinal sections were examined at ages P1, P3, P5, P7, P10, and P14. Adjacent to the immunolabeling are Nomarski images and intensity profiles. VGAT expression was first observed by $\mathrm{P} 1$ in the inner retina (Fig. 6A), and faint immunostaining was detected in the outer retina by P5-P7 (Fig. 6C,D). VGAT immunostaining had an adult pattern of expression by P10. A developmental series of VGAT immunostaining in the mouse retina was investigated at ages P1, P3, P5, P7, P10, and P14 (Fig. 7). VGAT expression was observed by $\mathrm{P} 1$ in the inner retina, and faint immunolabeling was detected by $\mathrm{P} 3$ in the outer retina (Fig. 7A). VGAT immunolabeling had an adult pattern of expression by P7. Taken together, these findings indicate that VGAT expression is present in the inner retina by $\mathrm{P} 1$, and that vesicular GABA/glycinergic transmission is possible at an early age.

\section{VGLUT1}

Our developmental studies in rat and mouse retina show that VGLUT1 has a delayed temporal onset of expression compared with VGAT and SV2; VGLUT1 expression is first observed in the outer retina followed by the inner retina.

Figure 8 shows VGLUT1 immunostaining at ages P1, P3, P5, $\mathrm{P} 7, \mathrm{P} 10$, and P14 in the rat retina. Nomarski images and intensity profiles are adjacent to the VGLUT1 immunostaining. VGLUT1 immunostaining was first observed by P5-P7 in the outer retina (Fig. $8 C, D$ ), and by $\mathrm{P} 7$ in the inner retina (Fig. $8 D$ ). VGLUT1 immunostaining acquires an adult-like pattern by P14 (Fig. 9F). At ages P7 and P10, VGLUT1 has a selective distribution to presumptive ON and OFF strata of bipolar terminals in the IPL (Figs.
VGLUT1 Developing Mouse Retina
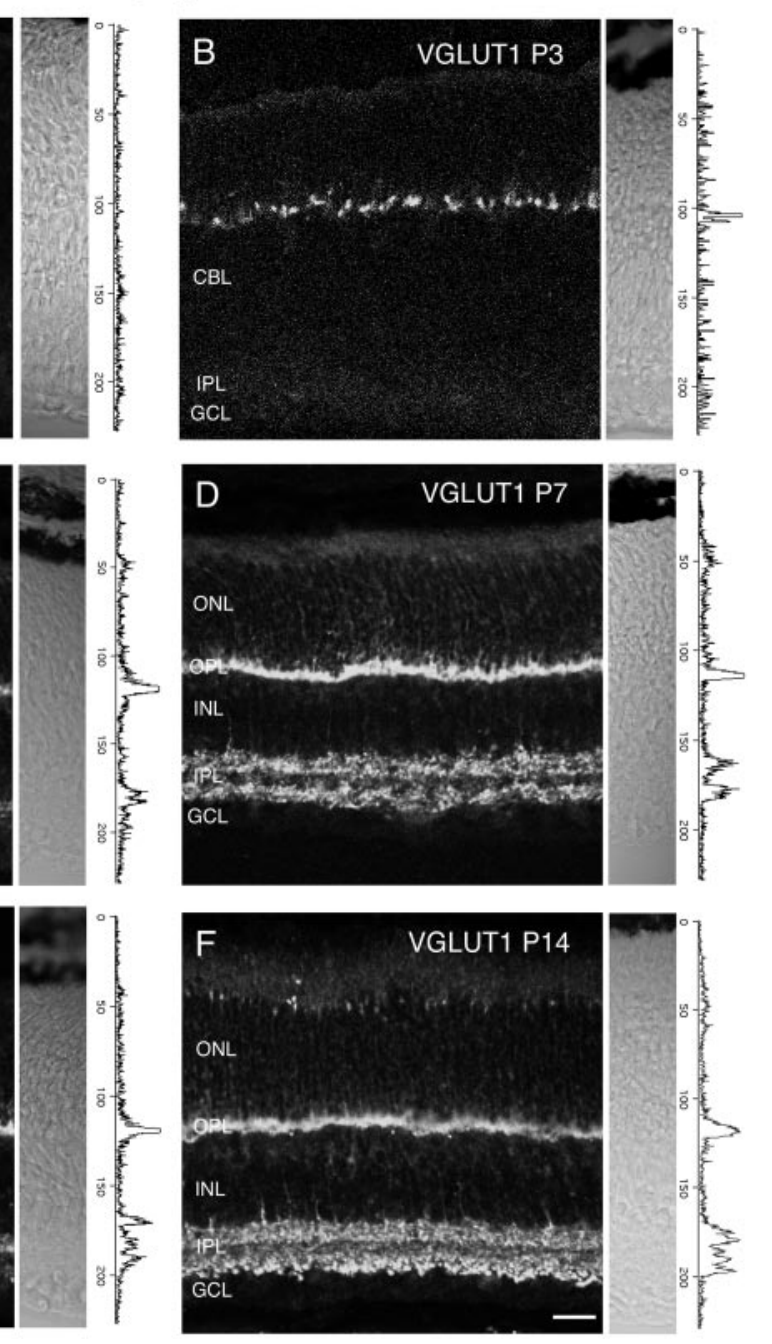
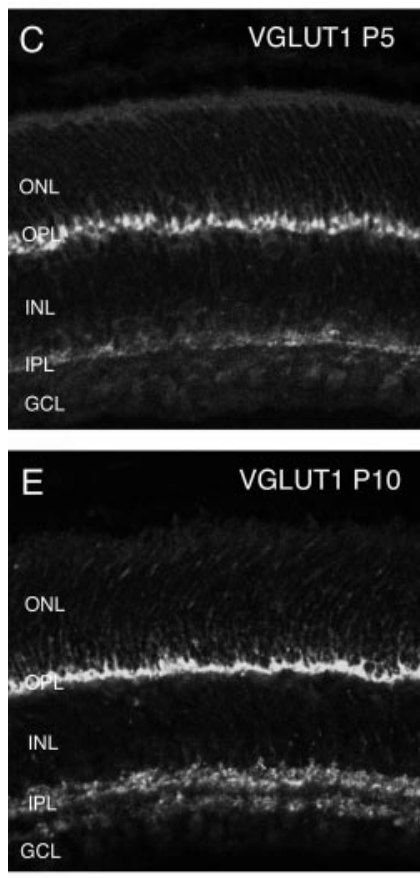

Figure 10. Vertical sections immunostained for VGLUT1 in the postnatal developing mouse retina. VGLUT1 expression was delayed compared with VGAT. VGLUT1 was first observed in the outer retina by P3 and the inner retina by P5. Retinal layers are shown with Nomarski optics adjacent to the micrographs. Intensity profiles are also included for each fluorescent image. VGLUT1 immunolabeling was examined at the following ages: $A, P 1 ; B, P 3 ; C, P 5 ; D, P 7 ; E, P 10 ; F, P 14$. Scale bar, $20 \mu \mathrm{m}$.

$8 A-F, 9)$. Figure 9 shows a $\mathrm{P} 7$ rat retina immunostained for VGLUT1 (Fig. 9A), and SV2 (Fig. 9B). The VGLUT1 stratification pattern in the IPL was not present in the SV2 immunostaining, which labeled the entire IPL (Fig. 9B). VGLUT1 expression was also examined in developing postnatal mouse retina at ages P1, P3, P5, P7, P10, and P14 (Fig. 10). VGLUT1 immunostaining was detected in the outer retina by $\mathrm{P} 3$ (Fig. $10 \mathrm{~B}, C$ ) and in the inner retina by P5 (Fig. 10C,D). An adult pattern of expression was found by P14. Our findings indicate that VGLUT1 expression is delayed in comparison with VGAT, and that vesicular glutamatergic transmission is possible by P7 in the inner retina of mouse and rat retina.

\section{SV2}

We examined SV2 expression in postnatal rat and mouse retina. We used this as an internal reference, because SV2 expression in developing rodent retina has been thoroughly investigated (Rich et al., 1997). A developmental series of SV2 immunostaining in rat retina at ages P1, P3, P5, P7, P10, and P14 is shown in Figure 11. Nomarski images and intensity profiles are adjacent to the SV2 immunostaining. In agreement with previous reports, SV2 expression was observed at early ages (Rich et al., 1997). SV2 


\section{SV2 Developing Rat Retina}
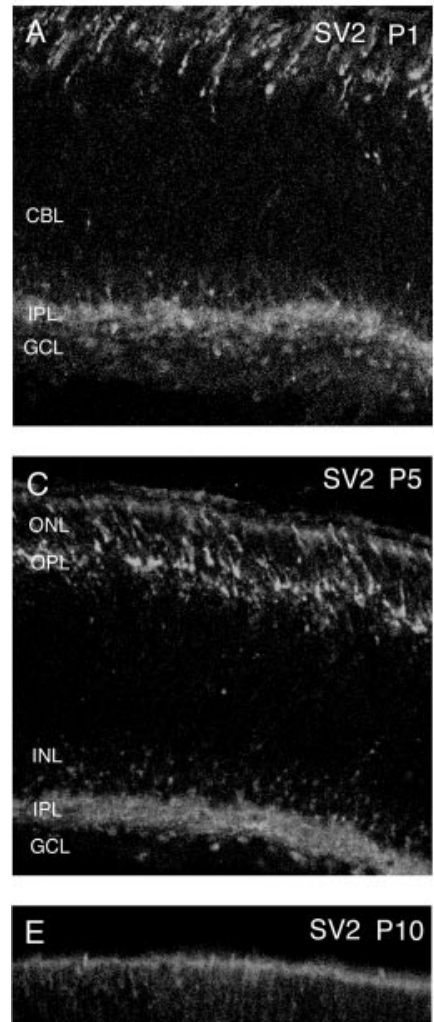

ONL

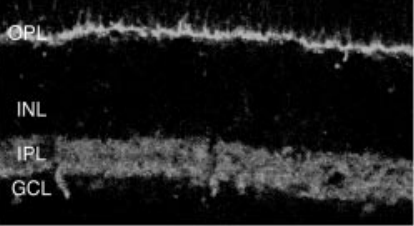

Figure 11. Vertical sections showing the postnatal development of SV2 in the rat retina. SV2 immunolabeling was present in the inner retina by P1. Retinal layers are shown with Nomarski optics adjacent to the micrographs. Intensity profiles are also included for each fluorescent image. SV2 immunolabeling was examined at the following ages: $A, P 1 ; B, P 3 ; C, P 5 ; D, P 7 ; E, P 10 ; F$, P14. Scale bar, $20 \mu \mathrm{m}$.

immunostaining was present in the inner retina by $\mathrm{P} 1$, and faint staining was observed in the outer retina at P1. Adult-like SV2 immunostaining was found by $\mathrm{P} 10$. We also observed expression of SV2 in developing postnatal mouse retina (Fig. 12) at ages P1, P3, P5, P7, P10, and P14. At some ages, we observed nonspecific immunostaining of blood vessels (Fig. 12D,F). Intense SV2 expression was present in the inner retina by $\mathrm{P} 1$ and in the outer retina by $\mathrm{P} 3$. An adult-like pattern was observed by $\mathrm{P} 7$. Taken together, these findings indicate that the synaptic vesicle protein SV2 is present in the inner retina of mouse and rat retina by $\mathrm{P} 1$.

\section{RIBEYE}

We examined expression of an antibody against RIBEYE in developing rat retina. Previous studies have found that RIBEYE is a component of the ribbon synapse (Schmitz et al., 2000). In agreement with previous reports, intense RIBEYE immunostaining was localized to the OPL, and discrete punctate immunolabeling was present throughout the IPL (Fig. 13F). In addition, we observed faint RIBEYE immunolabeling of cell bodies in the INL and GCL for all examined ages. Figure 13, $A$ and $B$, shows that RIBEYE immunoreactivity is not present in the outer or inner retina of $\mathrm{P} 5$ or $\mathrm{P} 7$ rat. Labeling for RIBEYE in the OPL was first observed at P10 (Fig. 13C). Faint labeling for RIBEYE was barely detected in the IPL at P10. More robust RIBEYE immunolabeling in the IPL was detected by P14 (Fig. 13D). RIBEYE immunostaining was present in the OPL and IPL of P15 and $\mathrm{P} 21$ rat retinas (Fig. $13 E, F$ ). Our findings indicate that RIBEYE expression is not present in the inner or outer retina of $\mathrm{P} 5$ and $\mathrm{P} 7$ rats.

In summary, these findings show that vesicular GABA/glycinergic transmission is possible in the inner retina by $\mathrm{P} 1$, whereas vesicular glutamatergic transmission has a delayed onset. VGLUT1 expression is observed at a time before ribbon structures are observed in bipolar cell terminals, suggesting that vesicular glutamatergic transmission occurs before the formation of ribbon synapses in bipolar cells.

Spontaneous excitatory and inhibitory synaptic events are observed in mouse retinal ganglion cells as early as $\mathrm{P7}$ We sought to determine whether sEPSCs to mouse retinal ganglion cells occur at an age when VGLUT1 expression is present but precedes the establishment of ribbon synapses in the inner retina. We examined spontaneous events to mouse retinal ganglion cells at ages P7-P9, P10-P12, and P13-P15. Previous work by our laboratory has investigated spontaneous excitatory and inhibitory synaptic inputs to retinal ganglion cells in mouse retina. These studies have found that histograms of the decay times of the spontaneous events allow for the separation of the events into two groups, a faster-decaying event group and a slower-decaying event group. The faster event group consists of AMPA-mediated glutamatergic sEPSCs, whereas the slower event group consists of GABA- and glycine-mediated sIPSCs (Tian et al., 1998; Tian and Copenhagen, 2001).

Figure $14 A$ shows a representative 5 min recording from a retinal ganglion cell of $\mathrm{P} 7$ mouse retina. The inset shows a single sEPSC and sIPSC on an expanded time scale. The rise time, peak amplitude, and monoexponential decay times of sEPSCs and sIPSCs were calculated from three groups of animals aged P7-P9, P10-P12, and P13-P16 (Fig. 14B). The average frequency (events per minute) of sEPSCs and sIPSCs increased with age. For example, at P7-P9, the average frequencies of sEPSCs and sIPSCs were $6.89 \pm 2.84$ and $8.18 \pm 2.81$ events/min, respectively. At P10-P12, the average frequencies of sEPSCs and sIPSCs increased to $23.1 \pm 7.01$ and $18.59 \pm$ 5.43 events/min, and by P13-P16, they were $32.79 \pm 8.19$ and $20.08 \pm 4.91$ events/min, respectively.

ANOVA tests were performed to determine statistically significant results. There was a significant difference in the average sEPSCs frequency between P7-P9 and P13-P16 aged mice (Fig. $14 C)(p=0.042$; ANOVA $)$. The average frequency of sIPSCs 
among the three groups of mice was not statistically significant. sEPSCs and sIPSCs were observed as early as P7. Our findings indicate the presence of vesicular glutamatergic and vesicular GABA and glycine neurotransmission as early as P7 in the inner retina of the mouse.

\section{Discussion}

We show here that VGLUT1 immunoreactivity in adult rat and mouse retina is found exclusively in the terminals of photoreceptor and bipolar cells, which are the principal glutamatergic neurons in the retina. VGLUT1 immunoreactivity colocalized with the synaptic vesicle marker SV2 to photoreceptor terminals in the OPL. VGLUT1 immunolabeling was found in a subset of SV2 synapses in the IPL, and VGLUT1 colocalized with PKC to rod bipolar terminals in the IPL. VGLUT1 also colocalized with LacZ to $\mathrm{ON}$ rod and cone bipolar terminals in mGlur6 ${ }^{+/-}$mice. The GABAergic marker GAD6 did not colocalize with VGLUT1, suggesting that VGLUT1 is not expressed in GABAergic synapses. VGLUT2, a vesicular glutamate transporter closely related to VGLUT1, was not expressed in glutamatergic synapses of photoreceptors or bipolar terminals. We conclude that VGLUT1 is the principal vesicular glutamate transporter used by photoreceptors and bipolar cells in the rodent retina.

We found that VGAT expression precedes VGLUT1 expression in postnatal mouse and rat retina. VGAT expression was present in the inner retina by $\mathrm{P} 1$ in mouse and rat retina, suggesting that vesicular GABA/glycinergic transmission is possible at an early age. We found sIPSCs in retinal ganglion cells as early as P7 in mouse retina. Furthermore, our studies demonstrate that VGLUT1 expression is present before the establishment of ribbon synapses in the inner retina of mouse (Fisher, 1979; Bachman and Balkema, 1993) and rat (Horsburgh and Sefton, 1987), indicating that vesicular glutamatergic transmission precedes the formation of ribbon synapses. In support of this hypothesis, we found that sEPSCs in mouse retinal ganglion cells were present as early as P7.

\section{Localization of VGLUT1 in adult retina}

VGLUT1 was localized exclusively to photoreceptor and bipolar terminals. These cells have a specialized synapse called a ribbon synapse. Photoreceptors in the OPL and bipolar cells in the IPL release glutamate in a tonic manner, which is regulated by small graded changes in the presynaptic potential (von Gersdorff et al., 1996; Tachibana, 1999). Although recent studies support a difference in release properties and synaptic protein composition between ribbon synapses in the OPL versus the IPL, our findings show that VGLUT1-mediated vesicle filling is similar in photoreceptors and bipolarcells. Photoreceptor ribbon synapses are thought to have a low threshold of intracellular calcium concen-

\section{SV2 Developing Mouse Retina}
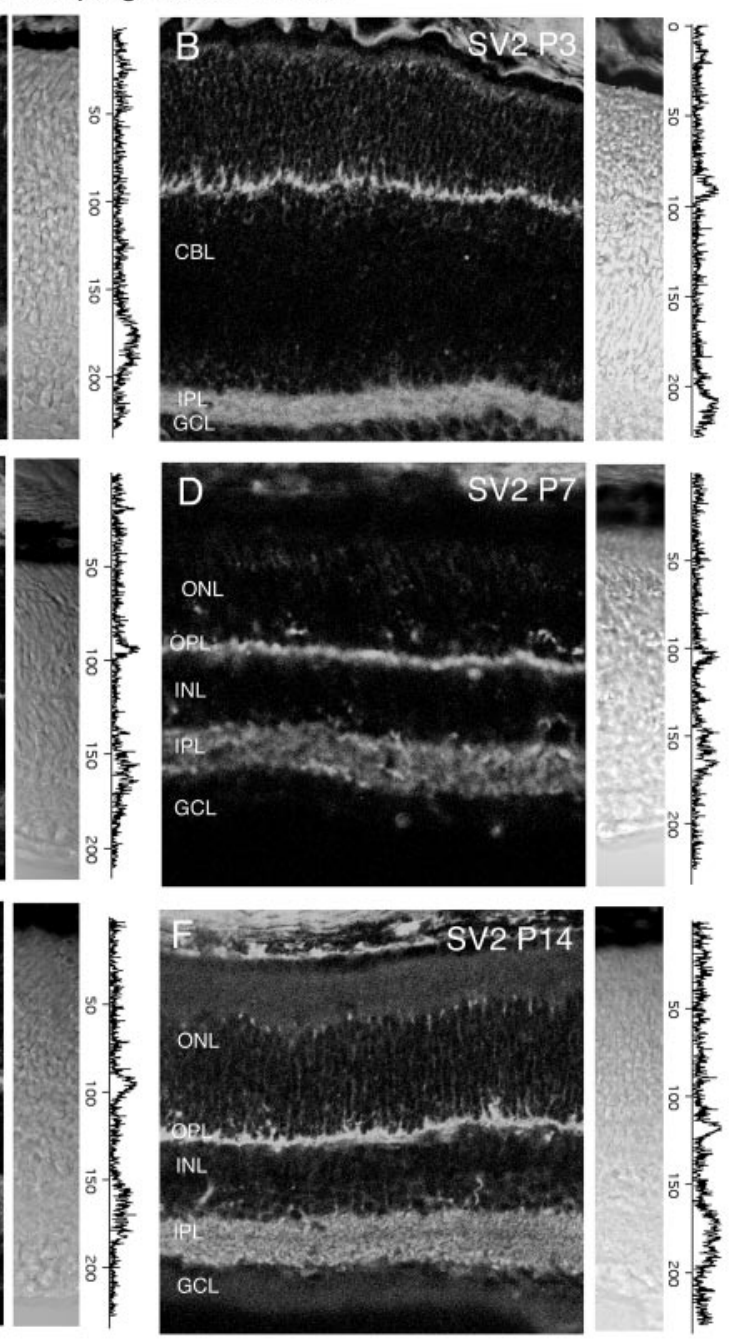

Figure 12. Vertical sections showing the postnatal development of SV2 in the mouse retina. SV2 had an early onset of expresIntensity profiles are also included for each fluorescent image. SV2 immunolabeling was examined at the following ages: $A, P 1 ; B$, $\mathrm{P} 3 ; C, \mathrm{P} 5 ; D, \mathrm{P} 7 ; E, \mathrm{P} 10 ; F, \mathrm{P} 14$. Scale bar, $20 \mu \mathrm{m}$.

tration for release, whereas bipolar ribbon synapses have a much higher threshold for release (von Gersdorff and Matthews, 1994; Rieke and Schwartz, 1996). Several synaptic proteins such as basson, postsynaptic density 95 (PSD-95), and PSD-93 have been found to differ between photoreceptors and bipolar cells (Koulen et al., 1998; Morgans, 2000a,b), supporting the hypothesis that expression of different synaptic protein components may account for distinct release properties. Our findings indicate that the mechanism for concentrating glutamate into vesicles in bipolar and photoreceptor terminals is the same, and that differences in release properties are not attributable to expression of distinct vesicular glutamate transporters.

\section{Developmental expression of VGAT and VGLUT1 in the retina}

VGAT and VGLUT1 expression was investigated in developing postnatal rat and mouse retina. In the rat and mouse retina, VGAT expression was observed at P1 in the inner retina. In the rat retina, VGLUT1 was found at P5-P7 in the outer retina and by $\mathrm{P} 7$ in the inner retina. In the mouse retina, VGLUT1 was observed at $\mathrm{P} 3$ in the outer retina and at P5 in the inner retina. 


\section{RIBEYE Developing Rat Retina}
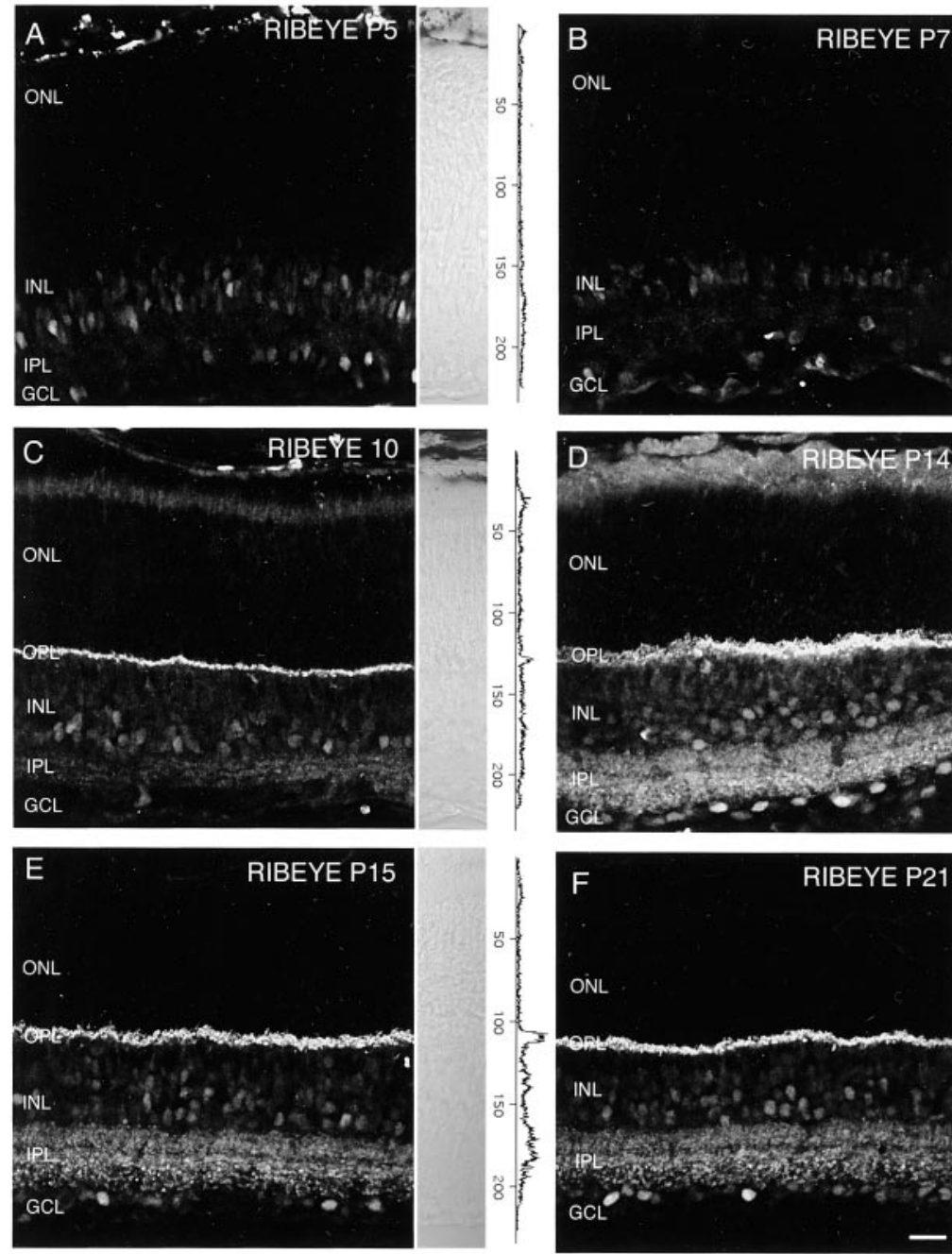

Figure 13. RIBEYE is not present in the inner rat retina by P7, an age at which there is strong immunostaining for VGLUT1. Vertical sections of postnatal rat retina immunostained for RIBEYE are shown. RIBEYE immunoreactivity is not present in the outer or inner retina of $\mathrm{P} 5$ or $\mathrm{P7}$ rat. Some nonspecific immunostaining of cell bodies was present in the INL and $\mathrm{GCL}$ at all ages examined. RIBEYE immunoreactivity is observed in the OPL of P10 rat retina, along with barely detectable immunostaining in the IPL. Robust immunostaining for RIBEYE was present in the OPL and IPL of P14 retina. RIBEYE localized to the OPL and IPL in P15 and P21 retina. Retinal layers are shown with Nomarski optics adjacent to the micrographs. Intensity profiles are also included for each fluorescent image. RIBEYE immunolabeling was examined at the following ages: $A, P 5 ; B, P 7 ; C, P 10 ; D, P 14 ; E, P 15 ; F$, P21. Scale bar, $20 \mu \mathrm{m}$.

\section{VGAT expression precedes VGLUT1 expression}

Our observation of VGAT expression in P1 inner retina indicates that vesicular GABA/glycinergic transmission is possible at this young age. In the mouse retina, conventional synapses in the inner retina are first detected at P3 (Fisher, 1979). VGAT colocalized with the synaptic vesicle marker SV2 in a subset of synapses in the IPL (Fig. 4C-E). VGAT did not colocalize with SV2 in the OPL (Fig. 4A), consistent with reports that VGAT is expressed in horizontal cells in the OPL (Cueva et al., 2002). The early appearance of VGAT, along with its colocalization with SV2, suggests that VGAT is localized to conventional synapses in the inner retina.

GABA is thought to have a neurotrophic role in the developing retina. For example, addition of $\mathrm{GABA}_{\mathrm{A}}$ receptor antagonists to rabbit explant cultures disrupts cone photoreceptor synaptogenesis (Huang et al., 2000). In young chick retina, GABA antagonists increase spontaneous waves (Wong et al., 1998), and in embryonic rabbit retina, GABA inhibits spontaneous waves
(Zhou, 2001). Our findings that VGAT is expressed in the inner retina by $\mathrm{P} 1$ suggests that vesicular GABAergic transmission occurs at a young age. Furthermore, we recorded sIPSCs onto retinal ganglion cells in P7 mouse retina. Previous studies have found GABAergic sIPSCs on rat ganglion cells as early as P5 (Rorig and Grantyn, 1993). In addition, GABA has been reported to have an excitatory action in young retina. GABA has a depolarizing effect in some ganglion cells of P2-P5 mouse retina (Bahring et al., 1994), and GABA application to neonatal rabbit retina (P0-P7) increases $\left[\mathrm{Ca}^{2+}\right]$ in all retinal layers (Huang and Redburn, 1996). It is believed that depolarizing GABA responses observed in young retinal neurons is attributable to lack of expression of neuron-specific $\mathrm{KCl}$ cotransporter 2 (KCC2). KCC2 expression increases in the second week of rat retinal development and is thought to act as a chloride extrusion system resulting in hyperpolarization in response to GABA ( $\mathrm{Vu}$ et al., 2000).

\section{VGLUT1 expression may precede formation of ribbon synapses in the inner retina}

VGLUT1 expression was present in the inner retina at an age when ribbon synapses are not yet observed. For example, VGLUT1 expression was present in P7 inner retina in the mouse and rat retina. In the mouse retina, electron microscopic studies have found that ribbon synapses are first detected at P11 (Fisher, 1979). Consistent with these studies, the ribbon synaptic marker B16 is first detected in mouse retina by $\mathrm{P} 13-\mathrm{P} 14$ in the inner retina (Bachman and Balkema, 1993). In the rat retina, electron microscopic studies have found that ribbon synapses are first detected in the inner retina by P13 (Horsburgh and Sefton, 1987). In addition, we found that RIBEYE, a component of the ribbon synapse, was first barely detected in the inner retina of rat at $\mathrm{P} 10$, with a more robust labeling in the inner retina at $\mathrm{P} 14$.

In agreement with our hypothesis that vesicular glutamate release precedes ribbon synapse formation in the inner retina, we found that sEPSCs in mouse retinal ganglion cells are present as early as P7 (Fig. 14). Interestingly, there was an increase with age in the average frequency of sEPSCs from mice aged P7-P9, P10$\mathrm{P} 12$, and P13-P16. There was a significant difference between the average frequency of sEPSCs of P7-P9 and P13-P16 mice. This increase in the average frequency of sEPSCs in the different age groups may correspond to the establishment of functional ribbon synapses, which may bolster the efficiency of vesicular glutamatergic transmission and result in an increased frequency of release. In agreement with our results, other studies have found glutamatergic inputs in young retina. For example, at P5 in rat 
retina, glutamatergic sEPSCs were observed in retinal ganglion cells (Rorig and Grantyn, 1993), whereas glutamatergic inputs were shown to influence spontaneous retinal waves in neonatal rabbit retina (Zhou and Zhao, 2000). Taken together, our findings indicate that vesicular glutamatergic transmission precedes ribbon synapse formation in developing postnatal rat and mouse retina.

\section{References}

Bachman KM, Balkema GW (1993) Developmental expression of a synaptic ribbon antigen (B16) in mouse retina. J Comp Neurol 333:109-117.

Bahring R, Standhardt H, Martelli EA, Grantyn R (1994) GABA-activated chloride currents of postnatal mouse retinal ganglion cells are blocked by acetylcholine and acetylcarnitine: how specific are ion channels in immature neurons? Eur J Neurosci 6:1089-1099.

Bellocchio EE, Hu H, Pohorille A, Chan J, Pickel VM, Edwards RH (1998) The localization of the brain-specific inorganic phosphate transporter suggests a specific presynaptic role in glutamatergic transmission. J Neurosci 18:86488659.

Bellocchio EE, Reimer RJ, Fremeau Jr RT, Edwards RH (2000) Uptake of glutamate into synaptic vesicles by an inorganic phosphate transporter. Science 289:957-960.

Buckley K, Kelly RB (1985) Identification of a transmembrane glycoprotein specific for secretory vesicles of neural and endocrine cells. J Cell Biol 100:1284-1294.

Chaudhry FA, Reimer RJ, Bellocchio EE, Danbolt NC, Osen KK, Edwards RH, Storm-Mathisen J (1998) The vesicular GABA transporter, VGAT, localizes to synaptic vesicles in sets of glycinergic as well as GABAergic neurons. J Neurosci 18:9733-9750.

Copenhagen DR, Jahr CE (1989) Release of endogenous excitatory amino acids from turtle photoreceptors. Nature 341:536-539.

Cueva JG, Haverkamp S, Reimer RJ, Edwards R, Wässle H, Brecha NC (2002) Vesicular $\gamma$-aminobutyric acid transporter expression in amacrine and horizontal cells. J Comp Neurol 445:227-237.

Euler T, Wässle H (1995) Immunocytochemical identification of cone bipolar cells in the rat retina. J Comp Neurol 361:461-478.

Fisher LJ (1979) Development of synaptic arrays in the inner plexiform layer of neonatal mouse retina. J Comp Neurol 187:359-372.

Fremeau Jr RT, Troyer MD, Pahner I, Nygaard GO, Tran CH, Reimer RJ, Bellocchio EE, Fortin D, Storm-Mathisen J, Edwards RH (2001) The expression of vesicular glutamate transporters defines two classes of excitatory synapse. Neuron 31:247-260.

Greferath U, Grunert U, Wässle H (1990) Rod bipolar cells in the mammalian retina show protein kinase C-like immunoreactivity. J Comp Neurol 301:433-442.

Haverkamp S, Grunert U, Wässle H (2000) The cone pedicle, a complex synapse in the retina. Neuron 27:85-95.
A

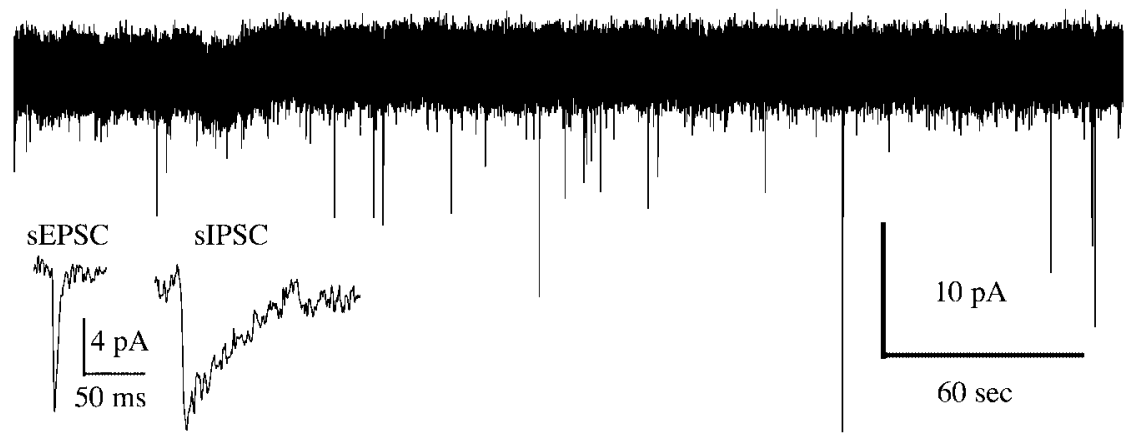

B

P7-9

$\mathrm{P} 10-12$

P13-16

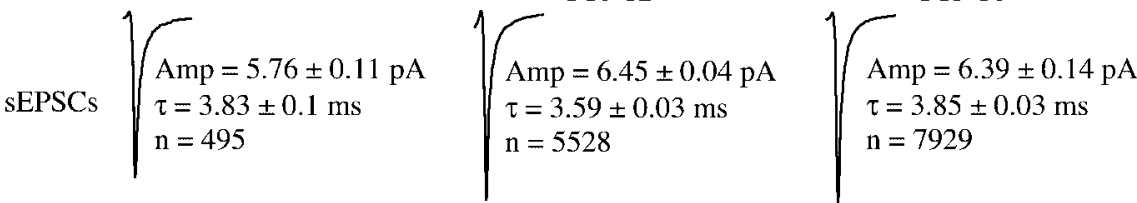

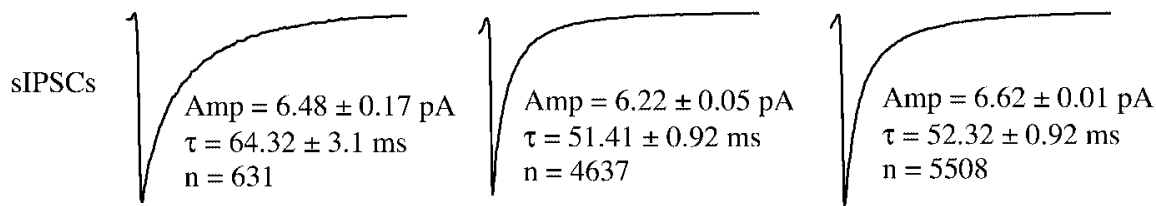

C
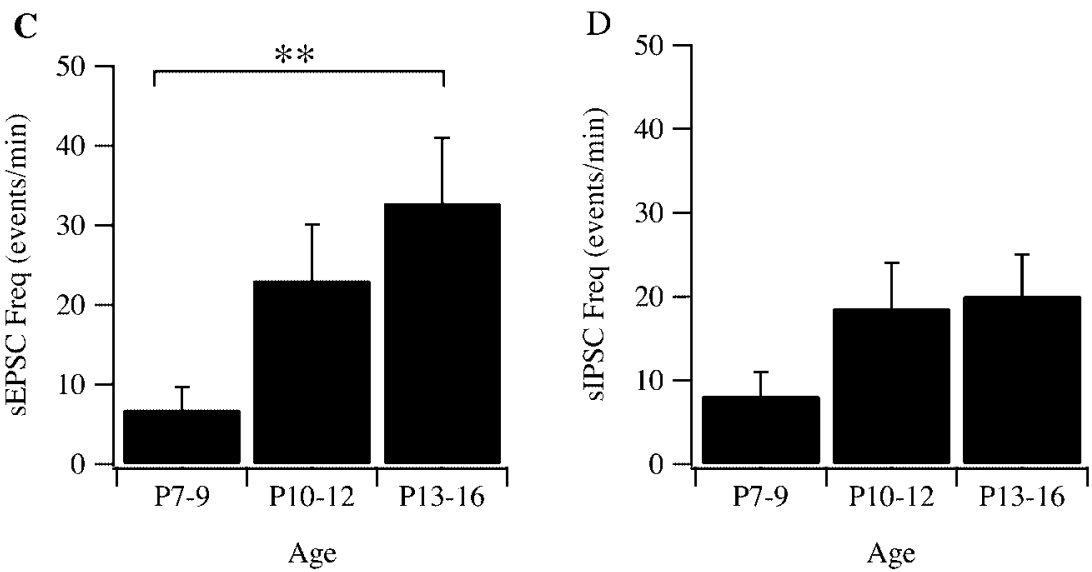

Figure 14. A, Representative 5 min recording of retinal ganglion cell membrane currents from a P7 mouse retina. Individual spontaneous events in the records were first detected and then quantified by the rise time, peak amplitude, and monoexponential decay times. In our previous studies, histograms of decay times showed a clear demarcation between a group of faster-decaying events and a group of slower-decaying events. The faster events were AMPA-mediated glutamatergic sEPSCs, and the slower ones were GABA- and glycine-mediated sIPSCs (Tian et al., 1998). Inset, Single sEPSC and sIPSC from the records. B, Average waveforms of $s$ EPSCs and sIPSCs from retinal ganglion cells of P7-P9, P10 -P12, and P13-P16 mice. Amp, Average amplitude of events \pm SE; $\tau$, monoexponential decay time constant of the averaged waveforms $\pm \mathrm{SE} ; n$, number of events for the calculation of each averaged waveform. ANOVA tests showed that the differences among the amplitudes of SEPSC $s$ from the three age groups were not statistically significant $(p=0.2817$ ). However, differences of the decay time constants between P7-P9 and P10-P12 and between $\mathrm{P} 10-\mathrm{P} 12$ and $\mathrm{P} 13-\mathrm{P} 15$ were significant ( $p=0.0289 ; p<0.0001$, respectively). ANOVA tests also showed that the difference of the sIPSC amplitudes between P10-P12 and P13-P15 mice was statistically significant ( $p=0.0005$ ). The differences of decay time constants between P7-P9 and P10 -P12 and between P7-P9 and P13-P15 were significant ( $p<0.0001$ ). $C$, Average frequency (events per minute) of retinal ganglion cell sEPSCs as a function of postnatal age. The difference of the average sEPSC frequency between P7-P9 and P13-P16 mice was statistically significant ${ }^{* *} p=0.042$; ANOVA). D, Average frequency (events per minute) of retinal ganglion cell sIPSCs as a function of postnatal age. The difference of the average sIPSC frequency among these three groups of mice was not statistically significant ( $p=0.371$; ANOVA). 
Horsburgh GM, Sefton AJ (1987) Cellulardegeneration and synaptogenesis in the developing retina of the rat. J Comp Neurol 263:553-566.

Huang B, Mitchell CK, Redburn-Johnson DA (2000) GABA and GABA(A) receptor antagonists alter developing cone photoreceptor development in neonatal rabbit retina. Vis Neurosci 17:925-935.

Huang BO, Redburn DA (1996) GABA-induced increases in $\left[\mathrm{Ca}^{2+}\right]_{\mathrm{i}}$ in retinal neurons of postnatal rabbits. Vis Neurosci 13:441-447.

Koulen P, Fletcher EL, Craven SE, Bredt DS, Wässle H (1998) Immunocytochemical localization of the postsynaptic density protein PSD-95 in the mammalian retina. J Neurosci 18:10136-10149.

Lee RY, Sawin ER, Chalfie M, Horvitz HR, Avery L (1999) EAT-4, a homolog of a mammalian sodium-dependent inorganic phosphate cotransporter, is necessary for glutamatergic neurotransmission in Caenorhabditis elegans. J Neurosci 19:159-167.

McIntire SL, Reimer RJ, Schuske K, Edwards RH, Jorgensen EM (1997) Identification and characterization of the vesicular GABA transporter. Nature 389:870-876.

Morgans CW (2000a) Neurotransmitter release at ribbon synapses in the retina. Immunol Cell Biol 78:442-446.

Morgans CW (2000b) Presynaptic proteins of ribbon synapses in the retina. Microsc Res Tech 50:141-150.

Ni B, Rosteck Jr PR, Nadi NS, Paul SM (1994) Cloning and expression of a cDNA encoding a brain-specific $\mathrm{Na}^{+}$-dependent inorganic phosphate cotransporter. Proc Natl Acad Sci USA 91:5607-5611.

Pothos EN, Larsen KE, Krantz DE, Liu Y, Haycock JW, Setlik W, Gershon MD, Edwards RH, Sulzer D (2000) Synaptic vesicle transporter expression regulates vesicle phenotype and quantal size. J Neurosci 20:7297-7306.

Rich KA, Zhan Y, Blanks JC (1997) Migration and synaptogenesis of cone photoreceptors in the developing mouse retina. J Comp Neurol 388:47-63.

Rieke F, Schwartz EA (1996) Asynchronous transmitter release: control of exocytosis and endocytosis at the salamander rod synapse. J Physiol 493:1-8.

Rorig B, Grantyn R (1993) Glutamatergic and GABAergic synaptic currents in ganglion cells from isolated retinae of pigmented rats during postnatal development. Brain Res Dev Brain Res 74:98-110.

Schmitz F, Konigstorfer A, Südhof TC (2000) RIBEYE, a component of synaptic ribbons: a protein's journey through evolution provides insight into synaptic ribbon function. Neuron 2000 28:857-872.

Tachibana M (1999) Regulation of transmitter release from retinal bipolar cells. Prog Biophys Mol Biol 72:109-133.
Tachibana M, Okada T (1991) Release of endogenous excitatory amino acids from ON-type bipolar cells isolated from the goldfish retina. J Neurosci 11:2199-2208.

Tagawa Y, Sawai H, Ueda Y, Tauchi M, Nakanishi S (1999) Immunohistological studies of metabotropic glutamate receptor subtype 6-deficient mice show no abnormality of retinal cell organization and ganglion cell maturation. J Neurosci 19:2568-2579.

Takamori S, Rhee JS, Rosenmund C, Jahn R (2000a) Identification of a vesicular glutamate transporter that defines a glutamatergic phenotype in neurons. Nature 407:189-194.

Takamori S, Riedel D, Jahn R (2000b) Immunoisolation of GABA-specific synaptic vesicles defines a functionally distinct subset of synaptic vesicles. J Neurosci 20:4904-4911.

Tian N, Copenhagen DR (2001) Visual deprivation alters development of synaptic function in inner retina after eye opening. Neuron 32:439-449.

Tian N, Hwang TN, Copenhagen DR (1998) Analysis of excitatory and inhibitory spontaneous synaptic activity in mouse retinal ganglion cells. J Neurophysiol 80:1327-1340.

von Gersdorff H, Matthews G (1994) Dynamics of synaptic vesicle fusion and membrane retrieval in synaptic terminals. Nature 367:735-739.

von Gersdorff H, Vardi E, Matthews G, Sterling P (1996) Evidence that vesicles on the synaptic ribbon of retinal bipolar neurons can be rapidly released. Neuron 16:1221-1227.

Von Kriegstein K, Schmitz F, Link E, Südhof TC (1999) Distribution of synaptic vesicle proteins in the mammalian retina identifies obligatory and facultative components of ribbon synapses. Eur J Neurosci 11:1335-1348.

Vu TQ, Payne JA, Copenhagen DR (2000) Localization and developmental expression patterns of the neuronal $\mathrm{K}-\mathrm{Cl}$ cotransporter (KCC2) in the rat retina. J Neurosci 20:1414-1423.

Wässle H, Yamashita M, Greferath U, Grunert U, Muller F (1991) The rod bipolar cell of the mammalian retina. Vis Neurosci 7:99-112.

Wong WT, Sanes JR, Wong RO (1998) Developmentally regulated spontaneous activity in the embryonic chick retina. J Neurosci 18:8839-8852.

Zhou ZJ (2001) A critical role of the strychnine-sensitive glycinergic system in spontaneous retinal waves of the developing rabbit. J Neurosci 21:5158-5168.

Zhou ZJ, Zhao D (2000) Coordinated transitions in neurotransmitter systems for the initiation and propagation of spontaneous retinal waves. J Neurosci 20:6570-6577. 